Check for updates

Cite this: RSC Adv., 2019, 9, 30398

\title{
Widely applicable phosphomolybdic acid doped poly(9-vinylcarbazole) hole transport layer for perovskite light-emitting devices
}

\author{
Yanting Wu, Zewu Xiao, Lihong He, Xiaoli Yang, Yajun Lian, Guoqing Li \\ and Xiaohui Yang (D)*
}

\begin{abstract}
In this paper, a cross-linked poly(9-vinylcarbazole) (PVK):phosphomolybdic acid (PMA) layer is used as the hole transport layer in perovskite light-emitting devices, and the morphology, crystal structure, and photophysical properties of perovskite films on the PVK:PMA layer are studied. The addition of PMA into the PVK layer improves the perovskite morphology integrity and promotes hole transport. As a result, perovskite light-emitting devices using a PVK:PMA hole transport layer exhibit an improved maximum luminous efficiency of $22.1 \mathrm{~cd} \mathrm{~A}^{-1}$ and power efficiency of $18.2 \mathrm{Im} \mathrm{W}^{-1}$ when compared with those of the counterparts with a PVK hole transport layer. Efficient perovskite light-emitting devices can be accessed by using various antisolvents due to the good solvent resistance of PVK:PMA networks. Moreover, the luminous efficiencies of perovskite light-emitting devices with a PVK:PMA hole transport layer are almost invariant irrespective of the presence of a hole injection layer, illustrating wide applicability of the PVK:PMA hole transport layer in perovskite light-emitting devices.
\end{abstract}

Received 24th July 2019

Accepted 13th September 2019

DOI: 10.1039/c9ra05734j

rsc.li/rsc-advances a maximum luminous efficiency of $15.9 \mathrm{~cd} \mathrm{~A}^{-1}$. We recently propose that addition of a UV ozone-modified organic interlayer between the cuprous thiocyanate (CuSCN) hole injection layer (HIL) and perovskite layer effectively suppresses perovskite luminescence quenching by CuSCN and simultaneously allows the subsequent layering of high quality perovskite films. ${ }^{19}$

Phosphomolybdic acid (PMA) is a kegging-type compound, which comprises a phosphorous anion and twelve molybdenum oxyanions attached by three protons. ${ }^{20-23}$ Quantum dot lightemitting devices containing a PMA HIL showed reduced operating voltage and improved luminous efficiency compared with the counterpart devices with a PEDOT:PSS HIL. ${ }^{22}$ PMA was shown to induce oxidative cross-linking of PVK, and resulting robust PVK:PMA networks with high resistance against the working solvents enabled preparation of solution-processed multilayer organic light-emitting devices with excellent performances. $^{23}$

Herein, we report preparation of good quality $\mathrm{MAPbBr}_{3}$ layers on the PVK:PMA layer. The addition of PMA into PVK layer promotes hole transport in light-emitting devices. $\mathrm{MAPbBr}_{3}$ light-emitting devices containing a PVK:PMA hole transport layer (HTL) show improved luminous efficiency and power efficiency when compared with the counterpart devices with a PVK HTL. Luminous efficiencies of $\mathrm{MAPbBr}_{3}$ lightemitting devices containing a PVK:PMA HTL are almost invariant irrespective of the presence of a HIL, illustrating wide applicability of PVK:PMA HTL in perovskite light-emitting devices. 


\section{Results and discussion}

\section{Characterization of PVK:PMA films}

We measure UV-vis absorption spectra of PVK:PMA films with different PMA concentrations annealed at $150{ }^{\circ} \mathrm{C}$ for $5 \mathrm{~min}$ and those of PVK:20\% PMA films annealed at different temperatures for 5 min (Fig. 1a and b). All samples exhibit a similar absorption profile with an absorption edge at $c a .350 \mathrm{~nm}$, mainly originating from PVK. ${ }^{24}$ PMA shows an absorption peak at $c a .320 \mathrm{~nm} \cdot{ }^{20}$ The absorbance of PVK:20\% PMA layers is not changed upon CB rinsing (Fig. 1c), indicating that the layers are fully cross-linked. The work function (WF) and ionization potential (IP) of the PVK layers have been determined to be $3.58 \mathrm{eV}$ and $5.98 \mathrm{eV}$ with ultraviolet photoelectron spectroscopy (Fig. 1d). Addition of PMA into the PVK layer increases the WF and IP to $4.17 \mathrm{eV}$ and $6.37 \mathrm{eV}$, respectively, implying that PVK layer is probably p-doped by PMA deduced from a larger WF shift with respect to the IP shift, ${ }^{20}$ which facilitates hole transport. ${ }^{25}$ AFM images of the PVK and PVK:20\% PMA samples annealed at $150{ }^{\circ} \mathrm{C}$ and the as-deposited PVK:20\% PMA samples are shown in Fig. $1 \mathrm{e}-\mathrm{g}$, respectively. All samples show a smooth surface with the similar root-mean-square (RMS) roughness values, indicating that the addition of PMA and thermal treatment have no significant effects on the HTL morphologies. Transmission Electron Microscopy (TEM) measurements reveal that the size of PMA clusters is 25-50 nm (Fig. 1h).

\section{Characterization of $\mathrm{MAPbBr}_{3}$ films}

Fig. 2a and b depict X-ray diffraction (XRD) patterns of $\mathrm{MAPbBr}_{3}$ films on the ITO/CuSCN/PVK:PMA ( $0 \%, 10 \%, 20 \%)$, and ITO/ CuSCN/PVK:20\% PMA (non-baked, baked at $100{ }^{\circ} \mathrm{C}$ or $150{ }^{\circ} \mathrm{C}$ ) substrates. All $\mathrm{MAPbBr}_{3}$ films show XRD diffraction peaks at
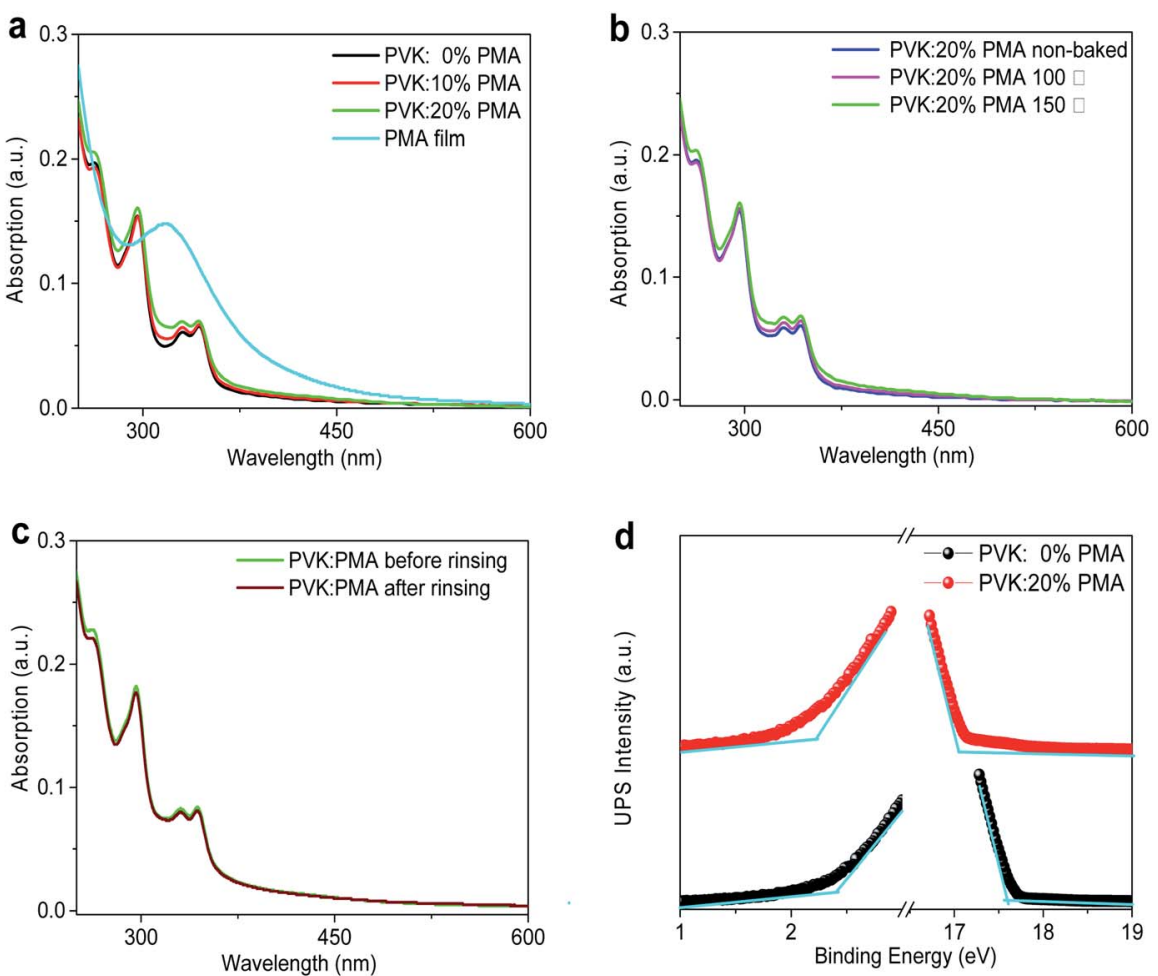

\section{PVK $150^{\circ} \mathrm{C}$}

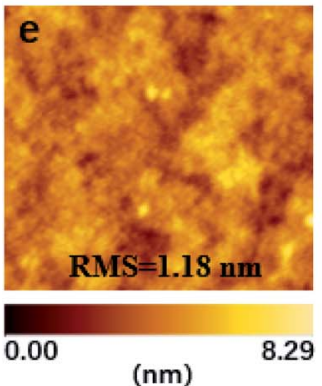

PVK:20\% PMA non-bake

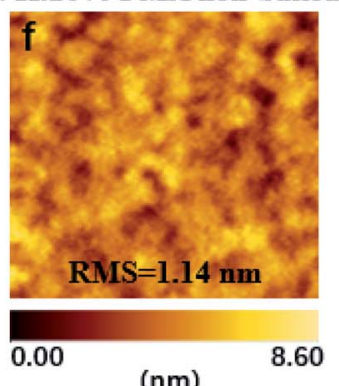

PVK:20\% PMA 150 C
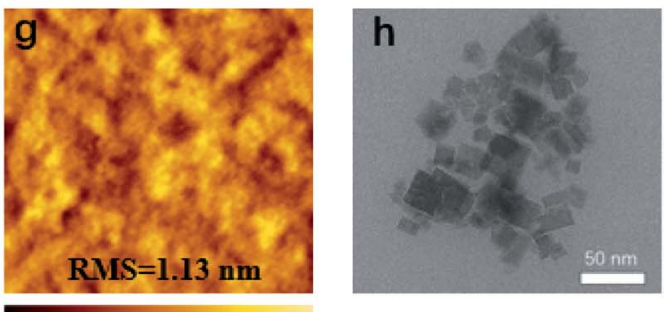

0.00

$(\mathrm{nm})$
7.53

Fig. 1 (a) UV-vis absorption spectra of PMA and PVK:PMA films with different PMA concentrations annealed at $150{ }^{\circ} \mathrm{C}$ for 5 min, (b) UV-vis absorption spectra of PVK:20\% PMA films annealed at different temperatures for $5 \mathrm{~min}$, (c) UV-vis absorption spectra of PVK:20\% PMA films and CB-rinsed PVK:20\% PMA films, and (d) secondary electron cut-off and valence band regions in UPS spectra of PVK and PVK:20\% PMA films. AFM images of (e) PVK:0\% PMA annealed at $150{ }^{\circ} \mathrm{C}$, PVK:20\% PMA non-annealed (f) and annealed at $150{ }^{\circ} \mathrm{C}(\mathrm{g})$. (h) TEM image of PVK:20\% PMA film. 

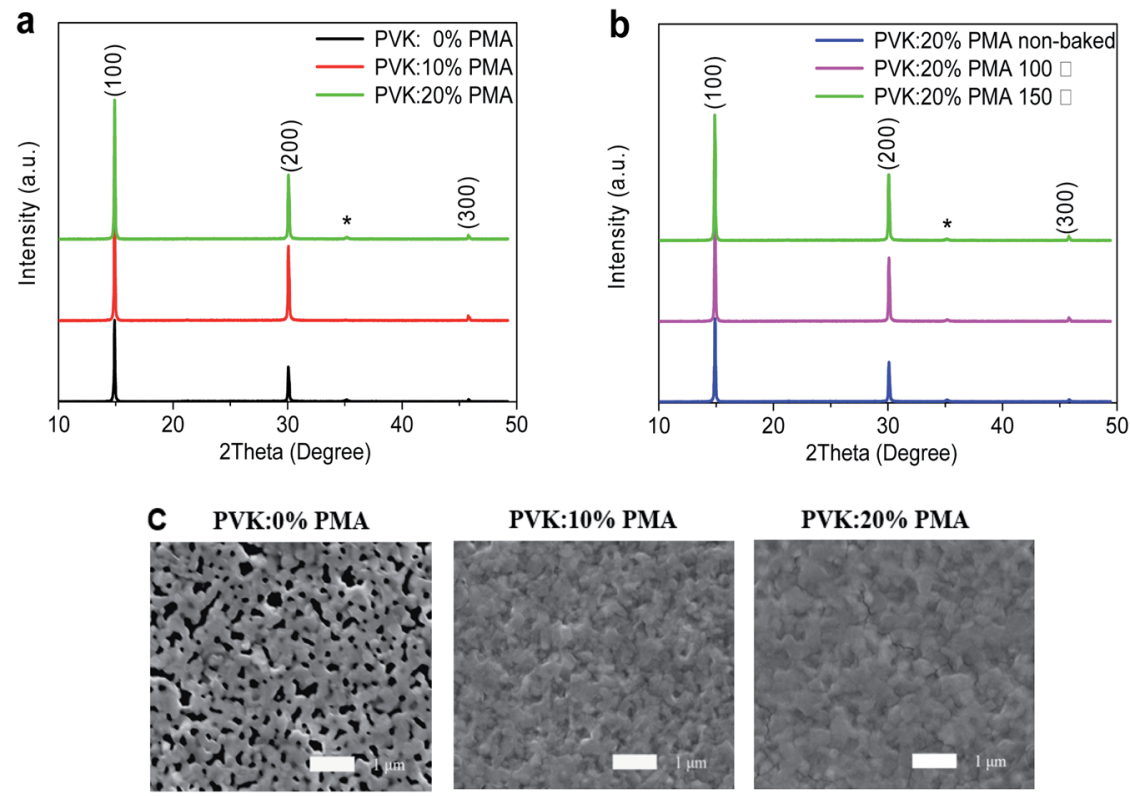

PVK:20\% PMA
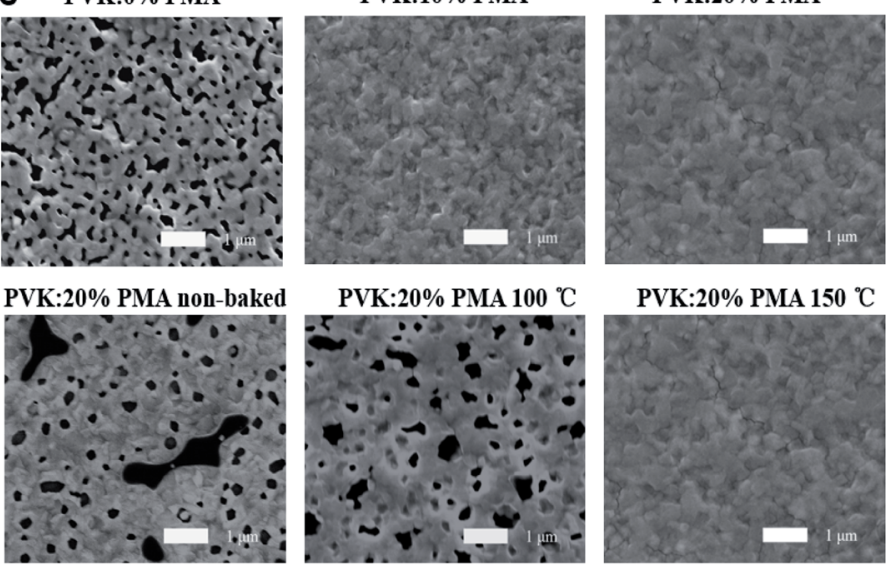

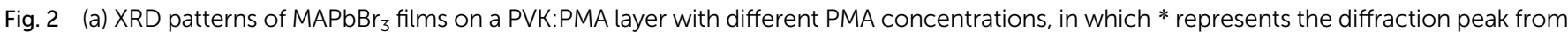
ITO. (b) XRD patterns of $\mathrm{MAPbBr}_{3}$ films on a PVK:20\% PMA layer annealed at different temperatures. (c) Top-view SEM images of MAPbBr 3 films on a PVK:PMA layer with different PMA concentrations and those on a PVK:20\% PMA layer annealed at different temperatures.

$14.9^{\circ}, 30.0^{\circ}$, and $45.8^{\circ}$, which are originated from the (100), (200), and (300) crystal planes of the $\mathrm{MAPbBr}_{3}$ cubic crystal structure, respectively. ${ }^{26}$ The (100) diffraction peak intensity increases upon the addition of PMA into the PVK layer and is not changed when the PMA concentration is increased from $10 \%$ to $20 \%$, indicating that incorporation of PMA slightly increases $\mathrm{MAPbBr}_{3}$ crystallinity. Similarly, the (100) diffraction peak intensity of the sample slightly increases following thermal treatment of PVK:20\% PMA layers at $100{ }^{\circ} \mathrm{C}$ and $150{ }^{\circ} \mathrm{C}$.

Top-view SEM images of $\mathrm{MAPbBr}_{3}$ films on the PVK:20\% PMA samples treated at different temperatures are shown in Fig. 2c. The substrate coverages of $\mathrm{MAPbBr}_{3}$ films increase with the increasing treatment temperature. $\mathrm{MAPbBr}_{3}$ films on the PVK:20\% PMA layer treated at $150{ }^{\circ} \mathrm{C}$ appear fully continuous and have negligible holes or cracks. The underlying reason for this is not clear at the present stage. We hypothesize it may relate to small amount of water in PMA sample. Ohisa et al. ${ }^{20}$ reported that the water content in PMA powder increased when stored in the ambient conditions. And water was reported to induce the change of $\mathrm{MAPbBr}_{3}$ crystallinity and the formation of cracks and pin-holes. ${ }^{27}$ Top-view SEM images of $\mathrm{MAPbBr}_{3}$ films on the PVK:PMA layers with different PMA concentrations treated at $150{ }^{\circ} \mathrm{C}$ are also shown in Fig. 2c. It appears that the PMA aids to improve the $\mathrm{MAPbBr}_{3}$ morphology integrity.
The PL maxima of all $\mathrm{MAPbBr}_{3}$ samples occur at $529 \mathrm{~nm}$, as shown in Fig. 3a and b. PL intensities of $\mathrm{MAPbBr}_{3}$ films decrease with increasing PMA concentration (Fig. 3a), which is possibly attributed to electron transfer from $\mathrm{MAPbBr}_{3}$ to PMA with a deep-lying WF of $6.4 \mathrm{eV} .^{20}$ Time-resolved PL decay plots of the $\mathrm{MAPbBr}_{3}$ samples are shown in Fig. 3c. All PL decay curves can be fitted with a double exponential function, where the fast and slow components correspond to trap-mediated nonradiative recombination process and radiative electron-hole bimolecular recombination process, respectively. ${ }^{28}$ The fitting parameters are provided in Table 1 . The average lifetime of $\mathrm{MAPbBr}_{3}$ films on the PVK layer (21.38 ns) is longer than those of $\mathrm{MAPbBr}_{3}$ films on the PVK:10\% PMA layer (11.68 ns) and PVK:20\% PMA layer (9.55 ns), which is in line with the steadystate PL measurement results.

In addition, as the annealing temperature for PVK:20\% PMA layers increases, PL intensity of the overlying $\mathrm{MAPbBr}_{3}$ layer is elevated (Fig. 3b), which may be associated with improved morphology integrity and reduced defect density in $\mathrm{MAPbBr}_{3}$ films.

\section{Light-emitting devices}

Light-emitting devices with the structure of ITO/CuSCN (15 nm)/ PVK:PMA (30 nm)/MAPbBr 3 (75 nm)/TmPyPB (60 nm)/CsF (1 nm)/ $\mathrm{Al}(100 \mathrm{~nm})$ are prepared to investigate the effects of PVK:PMA HTL incorporation on $\mathrm{MAPbBr}_{3}$ electroluminescent (EL) properties. 

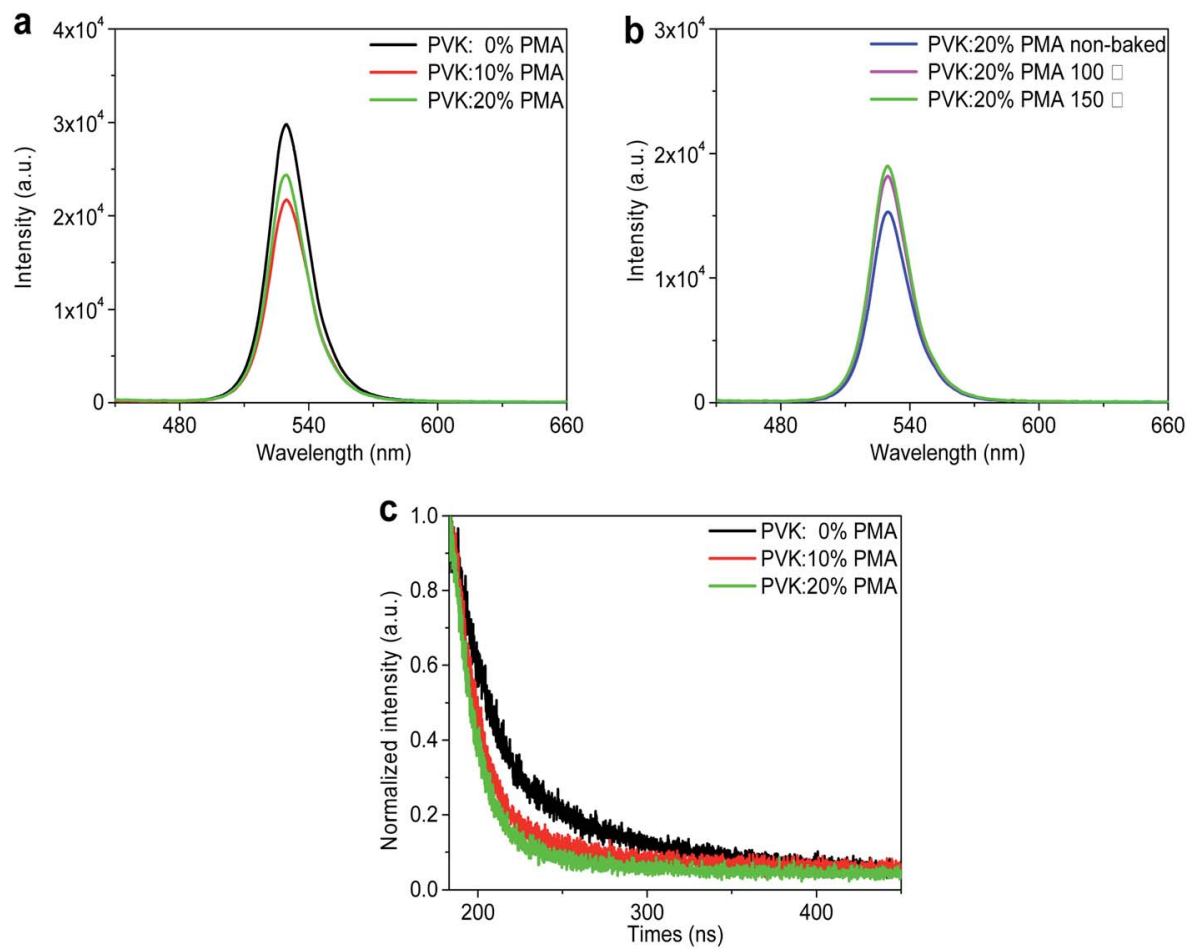

Fig. 3 (a) PL spectra of $M A P b B r_{3}$ films on the PVK:PMA layer with different PMA concentrations, (b) PL spectra of $M A P b B r_{3}$ films on the $P V K: 20 \%$ PMA layer annealed at different temperatures. (c) Time-resolved $\mathrm{PL}$ decay curves of $M A P b B r_{3}$ films on the PVK:PMA layer with different PMA concentrations

Schematic diagrams of the devices and their energy levels are shown in Fig. 4a and b. The inset shows a cross-sectional SEM image of a $\mathrm{MAPbBr}_{3}$ layer on the PVK:20\% PMA layer treated at $150{ }^{\circ} \mathrm{C}$. A compact $\mathrm{MAPbBr}_{3}$ layer with the thickness of $c a .70-100 \mathrm{~nm}$ forms a good contact with the PVK:PMA layer. Except the energy levels of PVK:PMA layers (Fig. 1d), the energy levels of other materials and

Table 1 Fitting parameters for the time-resolved PL decay curves of $\mathrm{MAPbBr}_{3}$ films on the PVK:PMA layer with different PMA concentrations

\begin{tabular}{lrlllr}
\hline & $\tau_{1}(\mathrm{~ns})$ & $A_{1}(\%)$ & $\tau_{2}(\mathrm{~ns})$ & $A_{2}(\%)$ & $\tau_{\text {ave }}(\mathrm{ns})$ \\
\hline PVK:0\% PMA & 10.44 & 72 & 49.81 & 28 & 21.38 \\
PVK:10\% PMA & 7.77 & 91 & 49.04 & 9 & 11.68 \\
PVK:20\% PMA & 6.89 & 92 & 41.25 & 8 & 9.55
\end{tabular}

work function of the electrodes are obtained from the literature., ${ }^{3,26,29}$ The $J-L-V$ and luminous efficiency-current density characteristics of the devices that contain a PVK:PMA HTL with different PMA concentrations are shown in Fig. 5a and b, respectively. Under certain voltage, current density of the devices increases with increasing PMA concentration, indicating that the addition of PMA into PVK layer promotes hole transport. Hole-dominated devices containing a PVK:20\% PMA layer show larger current compared with that of the counterpart devices containing a PVK layer, confirming enhanced hole transport in the devices containing a PVK:PMA HTL (Fig. 5c). Meanwhile, the maximum luminous efficiencies of the devices increase with increasing PMA concentration, which is mainly associated with improved $\mathrm{MAPbBr}_{3}$ morphology integrity. Among the devices, the PVK:20\% PMA device shows the highest maximum luminous efficiency of $22.1 \mathrm{~cd} \mathrm{~A}^{-1}$ and power efficiency of $18.2 \mathrm{~lm} \mathrm{~W}^{-1}$, respectively.

Table 2 Summarization of the metrics of light-emitting devices using a PVK:PMA HTL with different PMA concentrations or with a PVK:20\% PMA $\mathrm{HTL}$ treated at different annealing temperatures

\begin{tabular}{lccc}
\hline HTLs & $\mathrm{LE}_{\max }\left(\mathrm{cd} \mathrm{A}^{-1}\right)$ & $\mathrm{PE}_{\max }\left(\operatorname{lm~W}^{-1}\right)$ & $\begin{array}{l}\text { Luminance @ } \\
6 \mathrm{~V}\left(\mathrm{~cd} \mathrm{~m}^{-2}\right)\end{array}$ \\
\hline PVK:0\% PMA & 16.2 & 12.1 & 3324 \\
PVK:10\% PMA & 19.0 & 14.8 & 12330 \\
PVK:20\% PMA & 22.1 & 18.2 & 11600 \\
PVK:20\% PMA non-baked & 0.7 & 0.4 & 1178 \\
PVK:20\% PMA $100{ }^{\circ} \mathrm{C}$ & 2.8 & 1.7 & 4492 \\
PVK:20\% PMA $150{ }^{\circ} \mathrm{C}$ & 22.1 & 18.2 & 11600
\end{tabular}


a

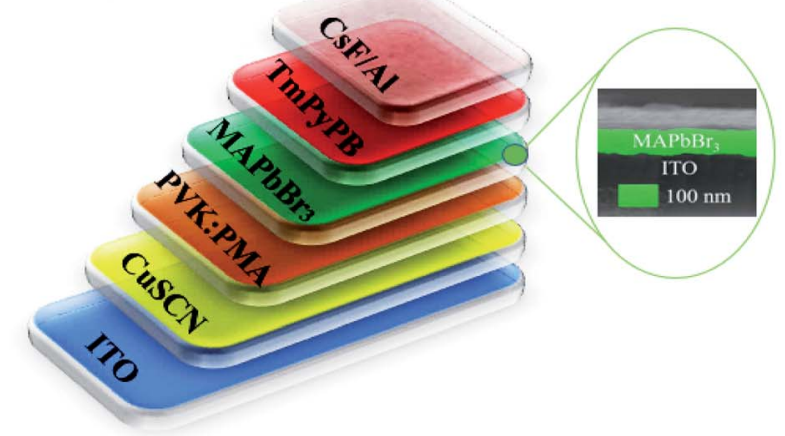

b

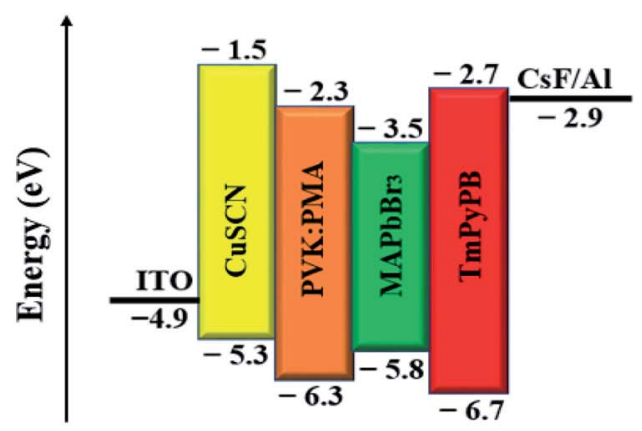

Fig. 4 (a) Schematic diagrams of the devices and (b) their energy levels. The inset shows a cross-sectional SEM image of a MAPbBr 3 layer on the CuSCN/PVK:20\% PMA bilayer pre-coated ITO substrate.

We fix the PMA concentration to $20 \%$ and investigate how the thermal treatment temperature for PVK:PMA layers affects the performance of perovskite light-emitting devices. The $J-L-V$ and luminous efficiency-current density characteristics of the devices are shown in Fig. $5 d$ and e. Compared with the devices containing a PVK:20\% PMA layer treated at $150{ }^{\circ} \mathrm{C}$, the devices with an asdeposited PVK:20\% PMA layer or with a PVK:20\% PMA layer treated at $100{ }^{\circ} \mathrm{C}$ exhibit inferior maximum luminous efficiencies of
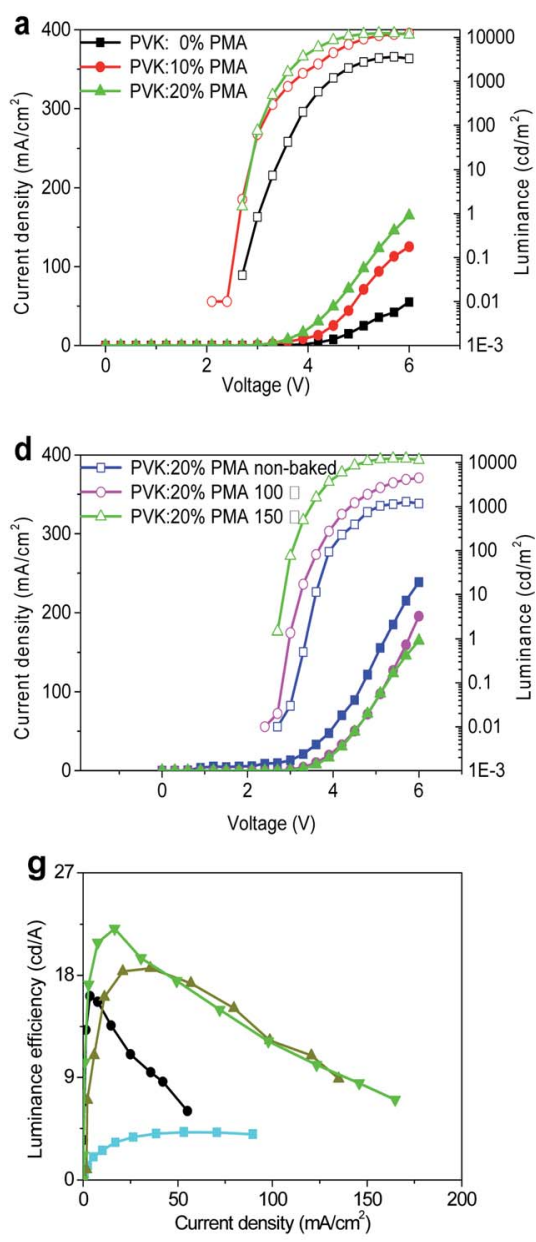
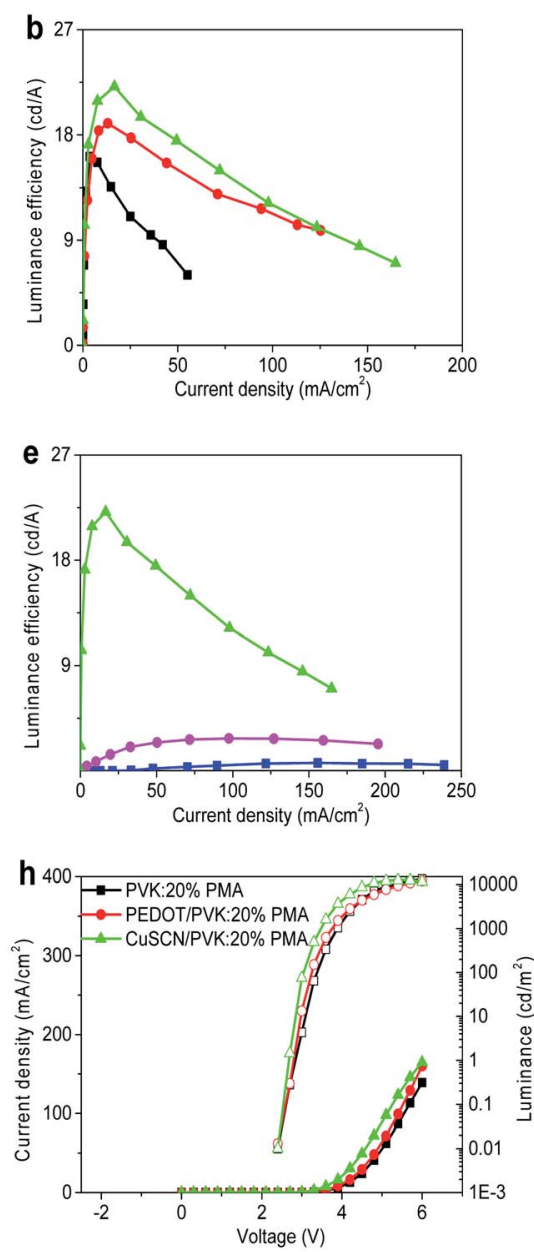
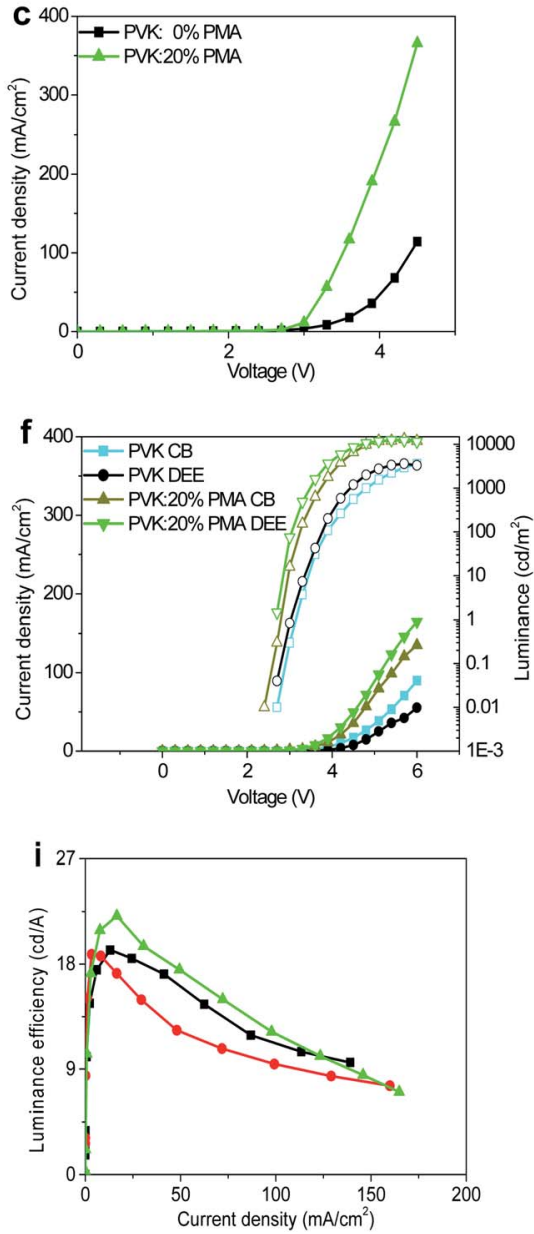

Fig. 5 (a) $J-L-V$ and (b) luminous efficiency-current density characteristics of light-emitting devices using a PVK:PMA HTL with different PMA concentrations. (c) $J-V$ characteristics of the hole-dominated devices with the structure of ITO/CuSCN/PVK or PVK:20\% PMA/MAPbBr $3 / \mathrm{mCP}$ Ag. (d) $J-L-V$ and (e) luminous efficiency-current density properties of light-emitting devices using a PVK:20\% PMA HTL annealed at different temperatures. (f) $J-L-V$ and (g) luminous efficiency-current density plots of light-emitting devices using a PVK:20\% PMA or PVK HTL processed with a CB or DEE antisolvent. (h) $J-L-V$ and (i) luminous efficiency-current density curves of light-emitting devices with different HILs. 

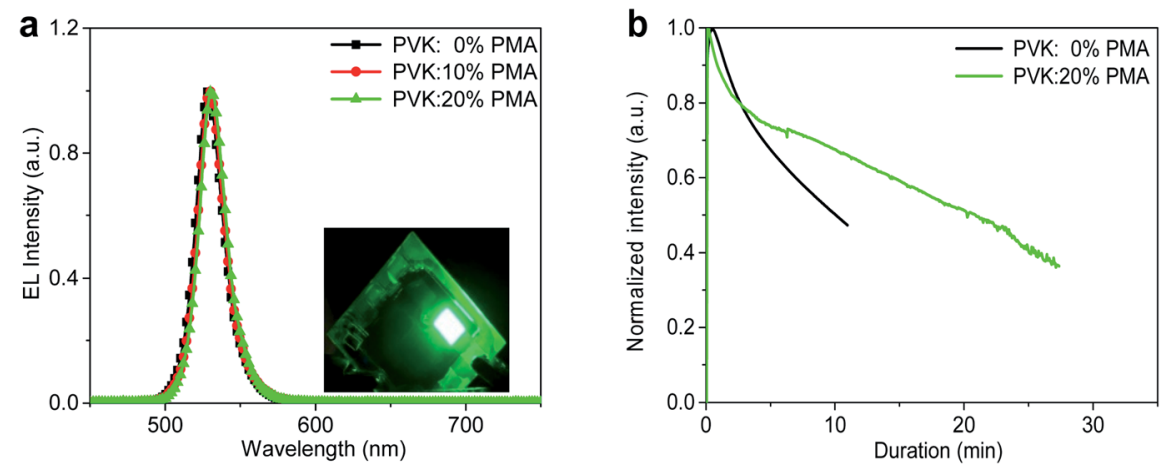

Fig. 6 (a) EL spectra of light-emitting devices using a PVK:PMA HTL with different PMA concentrations. The inset shows a photo of a lit device with a PVK:20\% PMA HTL. (b) Operational stability measurements of light-emitting devices with a PVK:20\% PMA or a PVK HTL under 3 mA cm ${ }^{-2}$.

$0.7 \mathrm{~cd} \mathrm{~A}^{-1}$ and $2.8 \mathrm{~cd} \mathrm{~A}^{-1}$, respectively, which may relate to the combinational effects of poor $\mathrm{MAPbBr}_{3}$ morphology integrity and high defect density in $\mathrm{MAPbBr}_{3}$ layers, as revealed by the SEM and steady-state PL measurements (Table 2).

Due to the cross-linking nature of PVK:PMA layers, properties of light-emitting devices processed with a $\mathrm{CB}$ or DEE antisolvent are similar, as shown in Fig. $5 \mathrm{f}$ and g. By contrast, DEE-processed lightemitting devices with a PVK HTL show much higher luminous efficiencies compared with the counterpart devices processed with CB due to damaging PVK layer by CB. The results indicate use of a PVK:PMA HTL allows the wide range antisolvent selection to optimize the properties of $\mathrm{MAPbBr}_{3}$ layers without disrupting device structure.

To further illustrate wide applicability of a PVK:PMA HTL, we prepare light-emitting devices devoid of HIL, with a PEDOT:PSS or CuSCN HIL, i.e. with the structure of ITO/HIL/PVK:20\% PMA (30 $\mathrm{nm}) / \mathrm{MAPbBr}_{3}(75 \mathrm{~nm}) / \mathrm{TmPyPB}(60 \mathrm{~nm}) / \mathrm{CsF}(1 \mathrm{~nm}) / \mathrm{Al}(100 \mathrm{~nm})$ and measure the $J-L-V$ and luminous efficiency-current density characteristics of light-emitting devices, which are shown in Fig. $5 \mathrm{~h}$ and $i$. The devices with a PVK:PMA HTL show similar luminous efficiencies irrespective of the presence of a HIL, which may relate to that PVK is p-doped by PMA, ${ }^{25}$ demonstrating wide applicability of PVK:PMA layer in perovskite light-emitting devices. Note that the devices with a CuSCN or PEDOT:PSS HIL show slightly larger current compared with the devices devoid of a HIL.

The devices containing a PVK:PMA HIL with different PMA concentrations all exhibit the characteristic $\mathrm{MAPbBr}_{3}$ emission with a maximum at $529 \mathrm{~nm}$ and a full width at half maximum (FWHM) value of $c a .20 \mathrm{~nm}$ (Fig. 6a), rendering a pure green emission with Commission Internationale de l'Eclairage (CIE) chromaticity coordinates of $(0.195,0.751)$. Light emission from the device with a PVK:20\% PMA HIL is uniform (Fig. 6a, inset). The operational stability measurements of the devices containing a PVK or PVK:20\% PMA HTL are shown in Fig. $6 \mathrm{~b}$. The operational lifetime $\left(\mathrm{T}_{50}\right)$ of the devices containing a PVK HTL, when driven at $3 \mathrm{~mA} \mathrm{~cm}$, is approximately $11 \mathrm{~min}$. Under the same driving conditions, the devices with a PVK:20\% PMA HTL exhibit a lifetime of $22 \mathrm{~min}$. Improved $\mathrm{MAPbBr}_{3}$ morphology integrity on a PVK:20\% PMA layer contributes to enhanced operational stability of light-emitting devices by reducing the shunt paths. Meanwhile, the addition of
PMA decreases operating voltage of light-emitting devices, and as a result may reduce the effect of Joule heating on the longevity of light-emitting devices. ${ }^{30}$

\section{Conclusion}

Addition of PMA into PVK layer aids to form good quality $\mathrm{MAPbBr}_{3}$ layer and facilitates hole transport, nevertheless, slightly quenches $\mathrm{MAPbBr}_{3}$ luminescence. Perovskite light-emitting devices that contain a PVK:PMA HTL exhibit a maximum luminous efficiency of $22.1 \mathrm{~cd} \mathrm{~A}^{-1}$ and power efficiency of $18.2 \mathrm{Im} \mathrm{W}^{-1}$, respectively, which are superior to those of the counterpart devices containing a PVK HTL. Use of a PVK:PMA HTL allows wide range antisolvent selection due to its cross-linking nature. In addition, the devices with a PVK:PMA HTL show similar luminous efficiencies irrespective of the presence of a HIL, demonstrating wide applicability of PVK:PMA layer in perovskite light-emitting devices.

\section{Experiment section}

\section{Materials}

Methylammonium bromide (MABr), lead(II) bromide ( $\left.\mathrm{PbBr}_{2}\right), 1,3,5-$ tri( $m$-pyrid-3-yl-phenyl)benzene (TmPyPB), and 1,3-bis( $N$-carbazolyl) benzene $(\mathrm{mCP})$ were purchased from Xi'an Polymer Technology (China). Poly(3,4-ethylenedioxythiophene):poly(styrenesulfonate) (PEDOT:PSS) (AI 4083) was obtained from Heraeus Corp. (Germany). CuSCN, dimethylformamide (DMF), chlorobenzene (CB), diethyl ether (DEE), and PVK were bought from Sigma-Aldrich (China). Diethyl sulfide (DES) was obtained from Shanghai Aladdin Industrial Corporation (China). Acetonitrile was purchased from Tianjin Kermel Chemical Testing Corp. (China). PMA was bought from Tokyo Chemical Industry Corp. All materials were used as received.

\section{Device fabrication}

CuSCN powder was dissolved in DES with a concentration of $20 \mathrm{mg} \mathrm{ml}^{-1}$ at $60^{\circ} \mathrm{C}$ for $1 \mathrm{~h}$. PVK and $\mathrm{mCP}$ were dissolved in CB with respective concentrations of 6 and $8 \mathrm{mg} \mathrm{ml}^{-1}$. PMA was dissolved in acetonitrile with a concentration of $5 \mathrm{mg} \mathrm{ml}^{-1}$. $\operatorname{MABr}(63 \mathrm{mg}, 0.53 \mathrm{mmol})$ and $\mathrm{PbBr}_{2}(137 \mathrm{mg}, 0.37 \mathrm{mmol})$ were 
dissolved into $1 \mathrm{ml}$ DMF. ITO substrates were sequentially cleaned with deionized water and organic solvents and afterward treated with UV ozone for $30 \mathrm{~min}$ immediately prior to device preparation. For preparation of a CuSCN HIL, the CuSCN solution was spin-coated onto the ITO substrates at $5000 \mathrm{rpm}$ for $60 \mathrm{~s}$. The CuSCN layers were heated at $60{ }^{\circ} \mathrm{C}$ for $3 \mathrm{~min}$. For preparation of a PEDOT:PSS HIL, the PEDOT:PSS water dispersion was spin-coated on the ITO substrates, which were thermally treated at $170{ }^{\circ} \mathrm{C}$ for $10 \mathrm{~min}$ to remove residual water. PVK and PVK:PMA HTLs were prepared by spin-coating the solutions at $3000 \mathrm{rpm}$ on the ITO substrates, PEDOT:PSS, or CuSCN layers. The PVK:PMA layers were subsequently treated at different temperatures for $5 \mathrm{~min}$. The $\mathrm{MAPbBr}_{3}$ precursor solution was spin-coated at $3000 \mathrm{rpm}$. During the film formation process, $400 \mu \mathrm{l}$ DEE or CB was dropped onto the spinning substrate to accelerate crystallization. The thickness of the $\mathrm{MAPbBr}_{3}$ layer was $c a .75 \mathrm{~nm}$ as determined from the crosssectional SEM measurements. Light-emitting devices were completed by sequentially depositing TmPyPB (60 nm), CsF (1 $\mathrm{nm})$, and $\mathrm{Al}(100 \mathrm{~nm})$ under a vacuum of $10^{-4} \mathrm{~Pa}$ onto the $\mathrm{MAPbBr}_{3}$ layers. Hole-dominated devices were prepared by the successive deposition of $\mathrm{mCP}(15 \mathrm{~nm})$ and $\mathrm{Ag}(100 \mathrm{~nm})$ onto the $\mathrm{MAPbBr}_{3}$ layers. All preparation processes were conducted inside a glovebox with water and oxygen concentrations of 1 ppm except for deposition of PEDOT:PSS layers.

\section{Device characterization}

The device's current density-luminance-voltage $(J-L-V)$ characteristics were measured using a programmed Keithley 2400 source-measure unit and a Konica-Minolta chroma meter CS100A. Electroluminescent (EL) spectra were recorded with an Ocean Optics USB4000 UV-vis spectrometer. UV-vis absorption spectra were characterized with a Shimadzu UV-2600 spectrophotometer. Steady-state PL spectra and time-resolved PL decay curves were acquired with a Hitachi F4600 fluorophotometer using an excitation wavelength of $405 \mathrm{~nm}$. X-ray diffraction (XRD) patterns were measured with a Rigaku D/Max-B X-ray diffractometer equipped with a $\mathrm{Cu} \mathrm{K} \alpha$ radiation source. Morphologies of the samples were studied using a JSF-7100 scanning electron microscope (SEM) and a Hitachi atomic force microcope (AFM). Ultraviolet photoelectron spectroscopy (UPS) measurements were performed using an ESCALAB 250Xi system. Transmission electron microscopy measurements were carried out with a FEI Talos F200 scanning/transmission electron microscope.

\section{Conflicts of interest}

There are no conflicts to declare.

\section{Acknowledgements}

Financial support by the National Natural Science Foundation of China (Grant No: 11474232).

\section{References}

1 Z. K. Tan, R. S. Moghaddam, M. L. Lai, P. Docampo, R. Higler, F. Deschler, M. Price, A. Sadhanala, L. M. Pazos, D. Credgington, F. Hanusch, T. Bein, H. J. Snaith and R. H. Friend, Nat. Nanotechnol., 2014, 9, 687-692.

2 H. Cho, S. H. Jeong, M. H. Park, Y. H. Kim and T. W. Lee, Science, 2015, 350, 1222-1225.

3 S. A. Veldhuis, P. P. Boix, N. Yantara, M. J. Li, T. C. Sum, N. Mathews and S. G. Mhaisalkar, Adv. Mater., 2016, 28, 6804-6834.

4 L. N. Quan, F. P. G. de Arquer, R. P. Sabatini and E. H. Sargent, Adv. Mater., 2018, 30, 1801996.

5 S. D. Stranks and H. J. Snaith, Nat. Nanotechnol., 2015, 10, 391-402.

6 N. N. Wang, L. Cheng, R. Ge, S. T. Zhang, Y. F. Miao, W. Zou, C. Yi, Y. Sun, Y. Cao, R. Yang, Y. Q. Wei, Q. Guo, Y. Ke, M. T. Yu, Y. Z. Jin, Y. Liu, Q. Q. Ding, G. C. Xing, H. Tian, C. H. Jin, F. Gao, R. H. Friend, J. P. Wang and W. Huang, Nat. Photonics, 2016, 10, 699-704.

7 B. R. Sutherland and E. H. Sargent, Nat. Photonics, 2016, 10, 295-302.

8 Y. Cao, N. Wang, H. Tian, J. Guo, Y. Wei, H. Chen, Y. Miao, W. Zou, K. Pan, Y. He, H. Cao, Y. Ke, M. Xu, Y. Wang, M. Yang, K. Du, Z. Fu, D. Kong, D. Dai, Y. Jin, G. Li, H. Li, Q. Peng, J. Wang and W. Huang, Nature, 2018, 562, 249-253.

9 K. Lin, J. Xing, L. N. Quan, F. P. G. de Arquer, X. Gong, J. Lu, L. Xie, W. Zhao, D. Zhang, C. Yan, W. Li, X. Liu, Y. Lu, J. Kirman, E. H. Sargent, Q. Xiong and Z. Wei, Nature, 2018, 562, 245-248.

10 S. Ahn, S. H. Jeong, T. H. Han and T. W. Lee, Adv. Opt. Mater., 2017, 5, 1600512.

11 Y. W. Zhu, Z. C. Yuan, W. Cui, Z. W. Wu, Q. J. Sun, S. D. Wang, Z. H. Kang and B. Q. Sun, J. Mater. Chem. A, 2014, 2, 1436-1442.

12 M. Jorgensen, K. Norrman and F. C. Krebs, Sol. Energy Mater. Sol. Cells, 2008, 92, 686-714.

13 H. Luo, X. Lin, X. Hou, L. Pan, S. Huang and X. Chen, NanoMicro Lett., 2017, 9, 39.

14 G. A. D. Benatto, B. Roth, M. V. Madsen, M. Hosel, R. R. Sondergaard, M. Jorgensen and F. C. Krebs, Adv. Energy Mater., 2014, 4, 1400732.

15 D. W. Zhao, M. Sexton, H. Y. Park, G. Baure, J. C. Nino and F. So, Adv. Energy Mater., 2015, 5, 1401855.

16 Y. K. Chih, J. C. Wang, R. T. Yang, C. C. Liu, Y. C. Chang, Y. S. Fu, W. C. Lai, P. Chen, T. C. Wen, Y. C. Huang, C. S. Tsao and T. F. Guo, Adv. Mater., 2016, 28, 8687-8694.

17 Z. B. Wang, Z. Luo, C. Y. Zhao, Q. Guo, Y. P. Wang, F. Z. Wang, X. M. Bian, A. Alsaedi, T. Hayat and Z. A. Tan, J. Phys. Chem. C, 2017, 121, 28132-28138.

18 L. Liu, Z. B. Wang, W. D. Sun, J. Zhang, S. Q. Hu, T. Hayat, A. Alsaedi, T. Hayat and Z. A. Tan, Chem. Commun., 2018, 54, 13283-13286.

19 Y. T. Wu, Z. W. Xiao, Y. J. Lian, X. F. Peng, L. L. He, X. L. Yang, Q. Wang, J. Ren, G. Q. Li and X. H. Yang, Org. Electron., 2019, 75, 105420. 
20 S. Ohisa, S. Kagami, Y. J. Pu, T. Chiba and J. Kido, ACS Appl. Mater. Interfaces, 2016, 8, 20946-20954.

21 M. Chrzanowski, M. Banski, P. Sitarek, J. Misiewicz and A. Podhorodecki, RSC Adv., 2019, 9, 10754-10759.

22 D. Dong, L. Lian, H. Wang and G. F. He, Org. Electron., 2018, 62, 320-326.

23 N. Aizawa, Y. J. Pu, T. Chiba, S. Kawata, H. Sasabe and J. Kido, Adv. Mater., 2014, 26, 7543-7546.

24 C. Zhang, H. Von Seggern, K. Pakbaz, B. Kraabel, H. W. Schmidt and A. J. Heeger, Synth. Met., 1994, 62, 35-40. 25 G. F. He, K. Leo, M. Hofmann, J. Birnstock, R. Pudzich and J. Salbeck, Appl. Phys. Lett., 2004, 85, 3911-3913.
26 X. F. Peng, X. Y. Wu, X. X. Ji, J. Ren, Q. Wang, G. Q. Li and X. H. Yang, J. Phys. Chem. Lett., 2017, 8, 4691-4697.

27 H. R. Wang, X. M. Li, M. J. Yuan and X. Y. Yang, Small, 2018, 14, 1703410.

28 J. Z. Chen and N. G. Park, Adv. Mater., 2018, 30, 1803019. 29 Q. Xiong, H. Tian, J. Zhang, L. Han, C. Y. Lu, B. H. Shen, Y. Zhang, Y. J. Zheng, C. J. Lu, Z. B. Zeng, Z. Y. Hu, L. H. Wu and Y. J. Zhu, Org. Electron., 2018, 61, 151.

30 Z. G. Xiao, R. A. Kerner, L. F. Zhao, N. L. Tran, K. M. Lee, T. W. Koh, G. D. Scholes and B. P. Rand, Nat. Photonics, 2017, 11, 108-115. 